\title{
Fermionic ground state at unitarity and Haldane Exclusion Statistics
}

\author{
R.K. Bhaduri ${ }^{1,2}$, M.V.N. Murthy ${ }^{1}$ and M. Brack ${ }^{3}$ \\ 1. The Institute of Mathematical Sciences, Chennai 600113, India \\ 2. Department of Physics and Astronomy, McMaster University, Hamilton, Canada L8S $4 M 1$ and \\ 3. Institute for Theoretical Physics, University of Regensburg, Regensburg, Germany
}

(Dated: October 30, 2018)

\begin{abstract}
We consider a few-particle system of trapped neutral fermionic atoms at ultra-low temperatures, with the attractive interaction tuned to Feshbach resonance. We calculate the energies and the spatial densities of the few-body systems using a generalisation of the extended Thomas-Fermi (ETF) method, and assuming the particles obey the Haldane-Wu fractional exclusion statistics (FES) at unitarity. This method is different from the scaled ETF version given by Chang and Bertsch (Phys. Rev. A76,021603(R) (2007)). Our semiclassical FES results are consistent with the Monte-Carlo calculations of the above authors, but can hardly be distinguished from their over all scaling of the ETF result at unitarity.

PACS numbers: PACS: 03.75.Ss, 05.30.-d
\end{abstract}

\section{INTRODUCTION}

There has been a lot of interest in a dilute gas of neutral fermionic atoms at ultra-cold temperatures both experimentally [1] and theoretically [2]. In general, the lowenergy properties of the gas are determined by the scattering length $a$, the number density $n$, and the temperature $T$ of the gas ( the effective range $r_{0}$ is small, so that $r_{0} /|a| \rightarrow 0$ as $a$ becomes large ). When the attractive interaction between the atoms is increased continuously by magnetic tuning from weak to strong, the scattering length $a$ goes from a small negative to a small positive value. In between, there is a zero-energy two-body bound state, and $|a|$ is infinite. The gas is said to be at unitarity in this situation, and the length scale $a$ drops out. The behaviour of the gas is expected to be universal at unitarity [2]. Understanding the ground state of the system in this limit is a challenge to many-body theorists as originally discussed by Bertsch [3]. Experimentally, if the temperature is small enough, a BCS superfluid is observed at the weak end, and a BEC condensate of dimers at the strong end [1]. This was predicted long back by Leggett [4], who extended the BCS formalism in a novel fashion to analyse the physical situation. The BCS to BEC transition is found to be smooth, with no discontinuity in properties across the unitary point.

Chang and Bertsch [5] have recently presented an $a b$ initio Green's Function Monte-Carlo (GFMC) calculation of the energy and density of $N=2-22$ trapped fermionic atoms in a harmonic potential. The atoms are interacting via a short range central two-body potential, with its strength adjusted to yield a zero-energy twobody bound state in free space. The many-body properties of this system are expected to be independent of the shape of the two-body interaction in such a set-up.

In an earlier paper [6] we obtained the energy per particle and the chemical potential of a noninteracting gas of atoms at finite temperatures obeying fractional exclusion statistics (FES) [7, 8]. We assumed that at unitarity, the effect of the interaction could be simulated by FES for the bulk properties of the system. Our results, with the choice of one free parameter in FES, were found to be in good agreement with theoretical MC calculations for a free gas 9, 10] and the experimental results in a trap [11, 12]. Since the number of atoms, $N$, was taken to be large, no finite- $\mathrm{N}$ corrections were needed in our semiclassical calculations. This is not the case in the present paper, where $N$ is taken to be small. The purpose of this paper is to test whether the FES hypothesis gives improved results when the finite-N corrections are incorporated in our calculations.

We briefly recall the rationale for using FES. As is well known, FES is realised by the Calogero-Sutherland model [13] in one dimension [14]. In two dimensions, the kinetic and potential energy densities of fermions interacting with a zero-range potential scale as the square of the spatial density, and obey FES in the mean-field approximation [15]. A hint that Haldane statistics is also realised for cold atoms in three dimensions at unitarity comes from the observation that the total energy per atom of the gas may be obtained by scaling the kinetic energy term by a constant factor [2]. This is however 
a necessary, but not a sufficient condition. A further hint comes from the fact that the second virial coefficient of the gas at unitarity is temperature independent [17]. In exclusion statistics, the scale-invariant interaction between atoms alters the ideal Fermi (Bose) values of the (exchange) second virial coefficient $+(-) 2^{-5 / 2}$ by adding an interacting part [16]. When FES is incorporated in the $T=0$ Thomas-Fermi (TF) method, it gives the same expression as the scaled density functional approach of Papenbrock 21]. This constitutes the bulk of the smooth part of the energy. It is the small next order term in the Extended Thomas-Fermi (ETF) expression that behaves differently with scaling or FES. The former was done by Chang and Bertsch [5]. In this paper, we incorporate FES in an improved version of ETF to test if it can differentiate between the two alternatives when compared with the GFMC results.

In the next section, we first summarise the TF and ETF results. Both these have limitations at the classical turning point, where the spatial density behaves discontinuously. To rectify this, we make use of a modified semiclassical method [18] that gives a continuous variation of the density across the turning point. Our semiclassical results incorporating FES are next compared with the GFMC calculations of Chang and Bertsch [5] for fermions trapped in a three dimensional oscillator potential. We find that FES results are consistent with the many-body GFMC results.

\section{SEMICLASSICAL CALCULATIONS INCORPORATING FES}

There has been much interest amongst theorists to calculate the properties of a gas in the unitary regime $\left(k_{f}|a|>>1\right)$, where $k_{f}=\left(3 \pi^{2} n\right)^{1 / 3}$ is the Fermi wave number of the noninteracting gas. This is a challenging task, since there is no small expansion parameter, and a perturbative calculation cannot be done. In particular, at $T=0$, the energy per particle of the gas is calculated to be $\frac{E}{N}=\xi \frac{3}{5} \frac{\hbar^{2} k_{f}^{2}}{2 M}$, where $\xi$ is a constant scaling factor [2]. A MC calculation gives $\xi \simeq 0.44[19]$. The experimental value is about 0.5 , but with large error bars [20]. A similar relation may be obtained assuming a noninteracting gas obeying FES, with a statistical occupancy factor $g$. At $T=0$, FES gives

$$
N=V \frac{1}{g} \frac{2}{(2 \pi)^{3}} \int_{0}^{\tilde{k_{f}}} 4 \pi k^{2} d k
$$

where we have included a spin degeneracy factor of 2 and the factor $1 / \mathrm{g}$ is the FES occupancy factor at $T=0$ with $g=1$ as the fermionic limit. The modified Fermi momentum $\tilde{k_{f}}$, from above. is $\tilde{k_{f}}=g^{1 / 3} k_{f}$, where $k_{f}$ is the fermi momentum of the noninteracting Fermi gas. It also follows that the energy per particle of the unitary gas is given by $\frac{E}{N}=g^{2 / 3} \frac{3}{5} \frac{\hbar^{2} k_{f}^{2}}{2 M}$. Comparing with the scaled version, we see that the scaling factor $\xi$ in a Fermi gas is related to the statistical parameter $g$ by the relation $\xi=g^{2 / 3}$. In a three-dimensional isotropic harmonic trap, a similar scaling of the TF expression gives 21], in units of $\hbar \omega$,

$$
E_{T F}=\frac{\xi^{1 / 2}}{4}(3 N)^{4 / 3} .
$$

In FES, an identical relation is obtained, with $\xi^{1 / 2}$ replaced by $g^{1 / 3}$. The scaled TF spatial density is also identical to the FES expression when this replacement is made :

$$
\rho_{T F}(r)=\frac{1}{3 \pi^{2} g}\left(\frac{2}{l^{2}}\right)^{3 / 2}\left((3 g N)^{1 / 3}-\frac{1}{2} \frac{r^{2}}{l^{2}}\right)^{3 / 2},
$$

where $l=\sqrt{(\hbar / m \omega)}$. The above expression is valid for $r \leq r_{0}$, where $r_{0}=\sqrt{2} l(3 g N)^{1 / 6}$ is the classical turning point. For $r>r_{0}$, the TF density is zero. To implement finite-N corrections, one has to consider ETF 22]. Chang and Bertsch [5] scale the energy expression for ETF by the same over all factor as in TF (denoted by ETF'), where as FES yields a different expression 23] (in units of $\hbar \omega)$ :

$$
\begin{aligned}
& E_{E T F}^{\prime}=\xi^{1 / 2}\left(\frac{(3 N)^{4 / 3}}{4}+\frac{(3 N)^{2 / 3}}{8}+\ldots\right) \\
& E_{E T F}=\left(g^{1 / 3} \frac{(3 N)^{4 / 3}}{4}+g^{-1 / 3} \frac{(3 N)^{2 / 3}}{8}+\ldots\right) .
\end{aligned}
$$

Although ETF gives a reasonable description of the smooth part of the energy, it fails to do so for the spatial density. In fact, the ETF density diverges at the turning point. To give a consistent description of both the energy and the spatial density, we adopt a method where a selective summation of the higher order gradient terms of the Wigner-Kirkwood series is made [18]. For a harmonic trapping potential $V(r)$, retaining terms up to third order in $\beta$, the Bloch density $C(r, \beta)$ incorporating FES is given by

$$
\begin{aligned}
C(r, \beta)= & \frac{1}{4 \pi^{2} g}\left(\frac{2 m}{\hbar^{2} \beta}\right)^{3 / 2} \\
& \left(1-\frac{\hbar^{2} \beta^{2}}{12 m}\right) \exp \left[-\beta V+\beta^{3} \frac{\hbar^{2}}{24 m}(\nabla V)^{2}\right](5)
\end{aligned}
$$


The spatial density is obtained by taking the inverse Laplace transform of $C(r, \beta) / \beta$ with respect to the chemical potential $\mu$, which we denote by $\tilde{\rho}=\mathcal{L}_{\mu}^{-1}[C(r, \beta) / \beta]$. It is the cubic term in the exponent that makes the density continuous across the classical turning point. Similarly, the energy is given by $\tilde{E}=\mu N-\mathcal{L}_{\mu}^{-1} C(r, \beta) / \beta^{2}$. The inverse Laplace transformations are carried out by the saddle-point method. The quality of the approximation is tested by applying the method to $N$ noninteracting spin-1/2 fermions $(g=1)$ in a harmonic potential. The result for the energy is plotted as a function of $N$ is plotted in Fig.1. To facilitate the comparison, the TF energy is subtracted out from the quantum as well as the semiclassical results for $E_{E T F}$ and $\tilde{E}$. Note that the shell effects in the energy as well as the density are not reproduced in the semiclassical calculations.

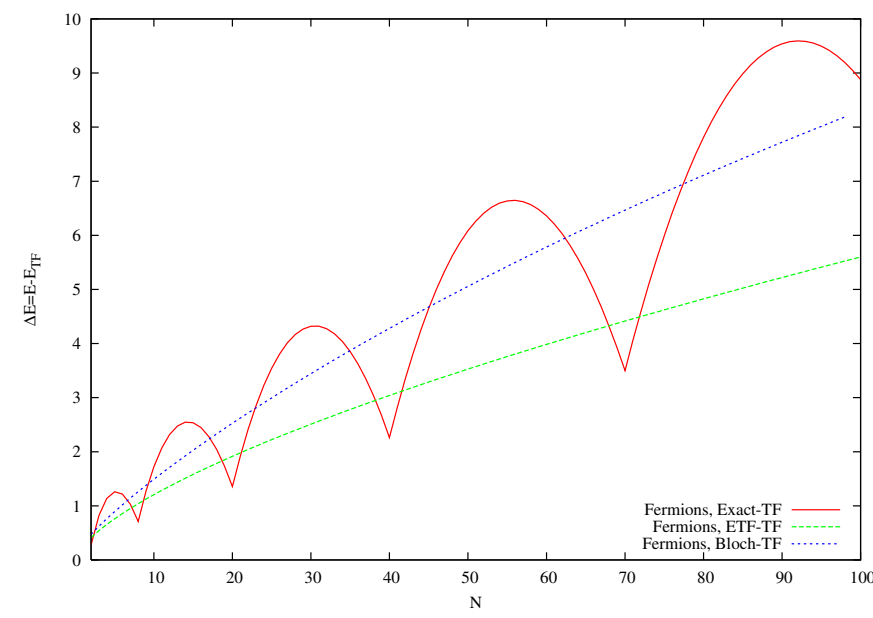

FIG. 1: Plot of the energy, $E-E_{T F}$ vs particle number for fermions (i.e., $g=1$ ). The red (solid) curve corresponds to the exact calculation in a harmonic oscillator while the green (dashed) and blue (dotted) curves correspond the calculations based on ETF and resummation methods.

In Fig.2, we compare the GFMC results [5] of the energy for $N=2-22$ atoms with the various semiclassical calculations. For the latter, the scaling factor in Eq.(3) is taken to be $\xi=0.48$, that corresponds to $g=1 / 3$ for the ETF Eq.(4), and also for $\tilde{E}$. Our choice of $\xi=0.48$ is very close to that of [5], and corresponds to a $g$ not too different from the value of 0.29 chosen earlier [6] . It is seen from Fig.2 that all the semiclassical methods fare well, and it is not possible to distinguish the scaled results from the FES ones. A clearer comparison is made in the inset of Fig.2, where the large TF term given by Eq.(1) is subtracted out from the energies. Even then, it is not possible to assert the relative superiority of over all scal-

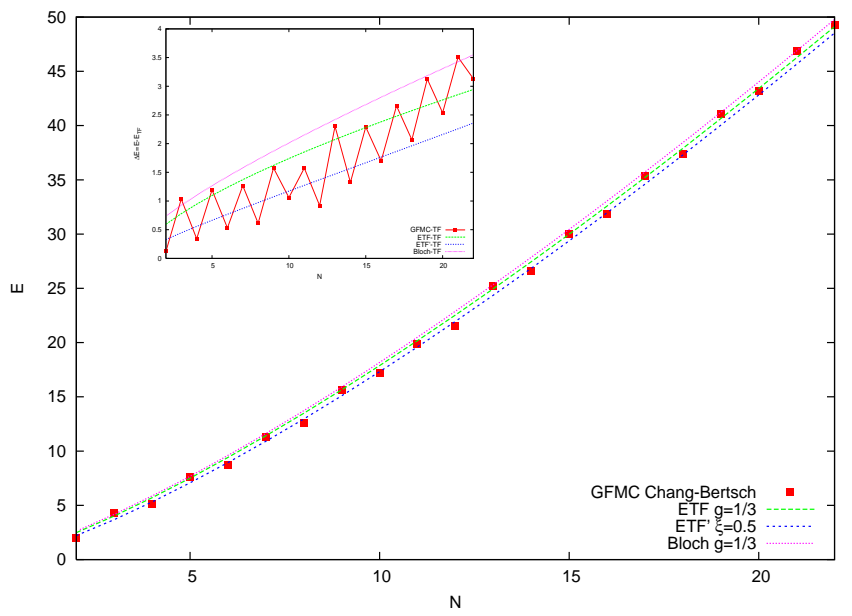

FIG. 2: Plot of the energy vs particle number with $g=1 / 3$. The data points refer to the GFMC calculation of Chang and Bertsch [5]. The pink(dotted) curve corresponds to the energy calculation using the resummation method. The green (dashed) and blue(short-dashed) curves correspond to the standard ETF calculation with FES (see Eq.(41)) and the ETF' approximation given by Chang and Bertsch (see eq.(3)). Inset shows the energy after subtracting the TF contribution as in Fig. 1 but choosing $g=1 / 3$.

ing to FES. We suggest that a distinction may possibly be made if a larger range of $N$ values are spanned by a MC many-body calculation. An interesting aspect of GFMC results (see inset in Fig.2) is the odd-even oscillations in energy. In Fig. 3 the calculated density for $N=20$ parti-

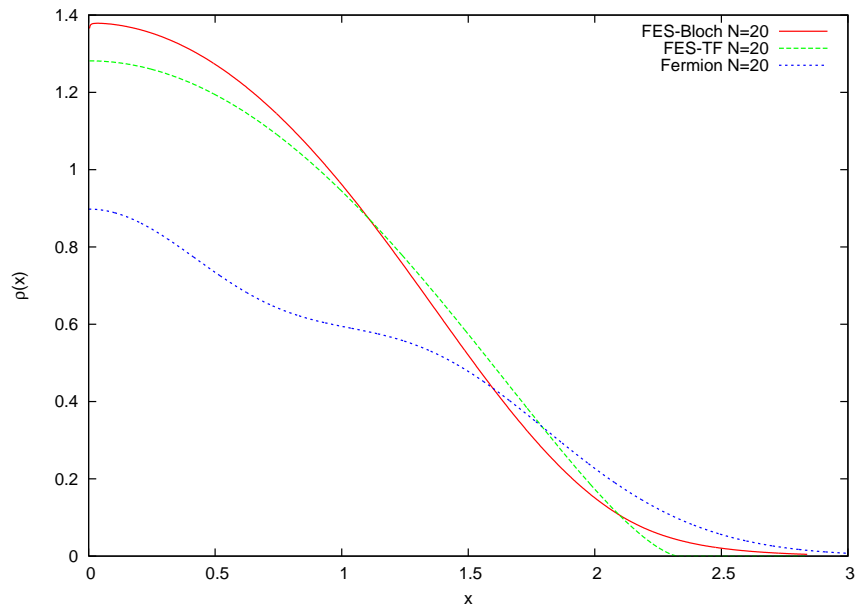

FIG. 3: Plot of radial density for $N=20$ as a function of the scaled distance $x$. Shown are the density calculated using resummation method (red-solid) and the TF density (greendashed) with $g=1 / 3$. The density of fermions in a harmonic oscillator is also shown for comparison (blue-dotted). 
cles is plotted using the resummation method and is compared with the TF density. Although there is not much to choose between the ETF and the resummation results for the energy, the density in the latter case is distinctly superior and appears to agree with the smoothed part of the density calculated by the the GFMC method. It also reproduces the tail beyond the turning point, which is not possible in TF or ETF approximations.

To summarise, We have considered a few particle system of trapped and interacting neutral fermionic atoms at ultra-low temperatures. The energy and spatial density of this system is calculated semiclassically assuming the particles obey the Haldane-Wu fractional exclusion statistics (FES) at unitarity. The semiclassical FES results are consistent with the Monte-Carlo calculations of Chang and Bertsch [5], but can hardly be distinguished from the over all scaling of the noninteracting energy that is commonly used at unitarity. However, it is interesting to note that both at finite temperature [6] and at zero temperature the FES frame work yields reasonably good results.

R.K.B. and M.V.N. acknowledge financial support of the Hans Vielberth University Foundation during their visits to Regensburg. M.B. acknowledges the hospitality of McMaster University and financial support by the NSERC.
[1] C.A. Regal et al., Nature (London) 424, 47 (2003); M.W. Zwierlein et al., Phys. Rev. Lett. 91, 250401 (2003); C.A. Regal et al., Phys. Rev. Lett. 92, 040403 (2004); M.W. Zwierlein et al., Nature (London) 435, 1046 (12005); G. B. Partridge et al., Science 311, 503 (2006).

[2] G.A. Baker, Phys. Rev. C60, 054311 (1999); H. Heiselberg, Phys. Rev. A63, 043606 (2001); T.-L. Ho. Phys. Rev. Lett. 92, 090402 (2004); G. Rupak, PRL (2006).

[3] G.F. Bertsch, "Many-body challenge problem" in Int. J. Mod. Phys. B15 (2001).

[4] A.J. Leggett, in Modern Trends in the Theory of Condensed Matter, Springer-Verlag Lecture Notes, Vol. 115, edited by A. Peklaski and J. Przystawa (Springer-Verlag, Berlin, 1980), p.13

[5] S.Y. Chang and G.F. Bertsch, Phys. Rev. A76, 021603 (2007)

[6] R.K. Bhaduri, M.V.N. Murthy and M. K. Srivastava, J.Phys.B: At.Mol.Opt.Phys.40, 1775 (2007)

[7] F.D.M. Haldane, Phys. Rev. Lett. 67, 937 (1991).

[8] A. Dasnieres de Veigy and S. Ouvry, Phys. Rev. Lett. 72, 600 (1994); S.B. Isakov, Mod. Phys. Lett. B8 319 (1994); Y.-S.Wu, Phys. Rev. Lett. 73, 922 (1994); A.K. Rajagopal, Phys. Rev. Lett. 74, 1048 (1995).

[9] A. Bulgac, J.E. Drut, and P. Magierski, Phys. Rev. Lett. 96, 090404 (2006).

[10] E. Burovski, N. Prokof'ev, B. Svistunov and M. Troyer, Phys. Rev. Lett. 96, 160402 (2006).

[11] J. Kinast et al. Science 3071296 (2005).

[12] H. Hu. Xia-Ji Lu and D. Drummond, Phys. Rev. A73, 023617 (2006).
[13] F. Calogero, J. Math. Phys. 10, 2191 (1969); 10, 2197 (1969); B. Sutherland, J. Math. Phys. 12. 246 (1971); 12, 251; Phys. Rev. A4, 2019 (1971).

[14] Z.N.C. Ha, Phys. Rev. Lett.73, 1574 (1994); S.B. Isakov, Phys. Rev. Lett. 73, 2150 (1994); M.V.N. Murthy and R. Shankar, Phys. Rev. Lett. 73, 3331 (1994).

[15] R.K. Bhaduri, M.V.N. Murthy, and M.K. Srivastava, Phys. Rev. Lett. 76, 165 (1996); R.K. Bhaduri, S.M. Reimann, S. Viefers, A. Ghose Choudhury and M.K. Srivastava, J. Phys. B: At. Mol.Opt.Phys. 33, 3895 (2000); T.H. Hansson, J.M. Leinaas, S. Viefers, Phys. Rev. Lett. 86, 2930 (2001).

[16] M.V.N. Murthy and R. Shankar, Phys. Rev. Lett. 72, 3629(1994).

[17] T-L Ho and E.J. Mueller Phys. Rev. Lett. 92, 160404 (2004).

[18] R. K. Bhaduri, Phys. Rev. Lett. 39, 329 (1977).

[19] J. Carlson, S.-Y. Chang, V.R. Pandharipande, and K.E. Schmidt, Phys. Rev. Lett. 91, 050401 (2003); A. Perali, P. Pieri, and G.C. Strinati, Phys. Rev. Lett. 93, 100404 (2004).

[20] M. Bartenstein et al., Phys. Rev. Lett. 92, 120401 (2004); T. Bourdel et al., Phys. Rev. Lett. 93, 050401 (2004).

[21] T. Papenbrock, Phys. Rev. A72, 041603 (2005).

[22] B. K. Jennings, R. K. Bhaduri and M. Brack, Nucl. Phys. A 253, 29 (1975); M. Brack and R. K. Bhaduri, Semiclassical Physics, ( Westview Press, 2003).

[23] B. P. van Zyl, D. A. W. Hutchinson and M. Need, cond-mat/0610256 